\title{
A Proposal to the Solution of Multiobjective Linear Fractional Programming Problem
}

\author{
Nuran Güzel \\ Department of Mathematics, Faculty of Art and Sciences, Yildiz Technical University, Davutpaşa, 34210 Istanbul, Turkey \\ Correspondence should be addressed to Nuran Güzel; nguzel@yildiz.edu.tr
}

Received 18 March 2013; Accepted 10 September 2013

Academic Editor: Mustafa Bayram

Copyright (C) 2013 Nuran Güzel. This is an open access article distributed under the Creative Commons Attribution License, which permits unrestricted use, distribution, and reproduction in any medium, provided the original work is properly cited.

\begin{abstract}
We have proposed a new solution to the Multiobjective Linear Fractional Programming Problem (MOLFPP). The proposed solution is based on a theorem that deals with nonlinear fractional programming with single objective function and studied in the work by Dinkelbach, 1967. As a new contribution, we have proposed that $\bar{x}$ is an efficient solution of MOLFPP if $\bar{x}$ is an optimal solution of problem $\operatorname{Max}_{x \in X} \sum_{i=1}^{k}\left(N_{i}(x)-Z_{i}^{*} D_{i}(x)\right)$, where is $Z_{i}^{*}=N_{i}\left(x_{i}^{*}\right) / D_{i}\left(x_{i}^{*}\right)$ for all $i$. Hence, MOLFPP is simply reduced to linear programming problem (LPP). Some numerical examples are provided in order to illustrate the applications of the proposed method. The optimization software package, namely, WinQSB (Chang, 2001), has been employed in the computations.
\end{abstract}

\section{Introduction}

Fractional programming concerns with the optimization problem of one or several ratios of functions subject to some constraints. These ratios are quantities that measure the efficiency of system, such as cost/profit, cost/time, cost/volume, and output/worker, while several ratios of functions are measured in different scales at the existence of some conflicts. The optimal solution for an objective function may not be an optimal solution for some other objective functions. Therefore, one needs to find the notion of the best compromise solution, also known as nondominant solution $[1,2]$.

In the literature, for various types of fractional programming, there are many different sorts of studies; some of them deal with theory [3-6], and some of them concern with solution methods [2, 7-18] and applications [19]. Dinkelbach [7] presented the algorithm based on a theorem by Jagannathan [20] concerning the relationship between fractional and parametric programming and restated and proved this theorem in somewhat simpler way. Leber et al. proposed [19] to use a fractional programming algorithm (the Dinkelbach algorithm) to calculate the melting temperature of pairings of two single DNA strands in biology.

If both the numerator and dominator of these ratios of functions in fractional programming are linear functions under some technological linear restrictions, then we have the multiple objective linear fractional programming (MOLFP) problems. There are so many studies including different approaches to solve different models of MOLFP problems in literature. Kornbluth and Steuer [21] proposed some possible linear fractional criteria [1] and have presented a generalized approach for solving a goal programming with linear fractional criteria [22]. Luhandjula [23] proposed a linguistic variable approach to solve a MOLFP problem. This approach simply and adequately describes imprecise aspirations of the decision maker to obtain a solution that is in some sense good in his/her opinion. These linguistic descriptions are considered as fuzzy objectives and are aggregated as in fuzzy linear programming [1].

Dutta et al. [24] modified the linguistic approach of Luhandjula such as to develop a method which yields always an efficient solution for optimising MOLFP problem. StancuMinasian and Pop [2] pointed out certain shortcomings in the work of Dutta et al. and have given the correct proof of theorem, which validates the obtaining of the efficient solutions. Lee and Tcha [25] developed iterative solution method to generate a sequence of linear inequality problems by parameterizing objective values to obtain a compromise solution of MOLFP problem. Chakraborty and Gupta [22] have presented a different methodology that always yields an efficient solution for solving MOLFP problem. In this 
methodology, MOLFPP may be solved easily with the transformation $y=t x, t>0$ resulting in a multiple objective linear programming (MOLP) problem. $t$ has been considered as the least value of both $1 / D_{i}(x)$ if objective function $Z_{i}(x) \geq 0$ for some $x$ in the feasible region and $1 /-N_{i}(x)$ if objective function $Z_{i}(x)<0$, for each $x$ in the feasible region. After original MOLFP problem reduces to an equivalent MOLP problem, the resulting MOLP problem is solved using fuzzy set theoretical approach by suitably defined membership functions and using min operator introduced by Zimmerman.

In this paper, we have investigated a solution to the MOLFP problem based on a theorem previously studied by Dinkelbach [7]. We have proposed that a feasible solution $\bar{x}$ of MOLFPP is an efficient solution of MOLFPP if $\bar{x} \in R^{n}$ is an optimal solution of problem $\operatorname{Max}_{x \in X} \sum_{i=1}^{k}\left(N_{i}(x)-Z_{i}^{*} D_{i}(x)\right)$, where is $Z_{i}^{*}=N_{i}\left(x_{i}^{*}\right) / D_{i}\left(x_{i}^{*}\right)$ for all $i$. Thus, MOLFPP is reduced to linear programming problem (LPP), and its solution procedure can be easily applied.

1.1. Linear Fractional Programming Problem (LFPP). The general LFPP is defined as follows:

$$
\underset{X \in x}{\operatorname{Maximize}} \frac{N(x)}{D(x)} .
$$

$N(x)=c^{T} x+\alpha, D(x)=d^{T} x+\beta$ are valued and continuous functions on $X$ and $d^{T} x+\beta \neq 0$ for all $X$ and $X=\{x \mid A x=$ $b, x \geq 0\}, x \in R^{n}, b \in R^{m}, A \in R^{n \times m}$.

$c^{T}, d^{T} \in R^{n}, \alpha, \beta \in R$ are assumed to be nonempty convex and compact set in $R^{n}$.

Theorem 1. Consider

$$
\begin{array}{ll}
\operatorname{Max} & \frac{N(x)}{D(x)} \\
\text { s.t. } & A x \leq b, \\
& x \geq 0, x \in X= \\
& \Longrightarrow D(x)>0 . \\
\text { Max } & t N\left(\frac{y}{t}\right) \\
\text { s.t. } & A\left(\frac{y}{t}\right)-b \leq 0, \\
& t D\left(\frac{y}{t}\right) \leq 1, \\
& t>0, y \geq 0 .
\end{array}
$$$$
x \geq 0, x \in X=\{x \mid A x=b, x \geq 0\}
$$

For some $\xi \in X, N(\xi) \geq 0$, if (2a) reaches a (global) maximum at $x=x^{*}$, then $(2 \mathrm{~b})$ reaches a (global) maximum at point $(t, y)=\left(t^{*}, y^{*}\right)$, where $y^{*} / t^{*}=x^{*}$ and the objective functions at these points are equal [22, 26].

Theorem 2 (see $[22,26])$. If $(2 \mathrm{a})$ is a standard concave-convex fractional programming problem which reaches a (global) maximum at point $x^{*}$, then the corresponding transformed problem (2b) attains the same maximum value at a point $\left(t^{*}, y^{*}\right)$, where $y^{*} / t^{*}=x^{*}$. Moreover $(2 \mathrm{~b})$ has a concave objective function and a convex feasible set.

Theorem 3 (see [7]). $z^{*}=N\left(x^{*}\right) / D\left(x^{*}\right)=\max \{N(x) / D(x) \mid$ $x \in X\}$ if and only if $F\left(z^{*}, x^{*}\right)=\max \left\{N(x)-z^{*} D(x) \mid x \in\right.$ $X\}=0$.

\section{Proposed Approach for Objective Functions of MOLFP Problem}

The vector-maximum Multiple Objective Linear Fractional programming (MOLFP) problem is defined as follows:

$$
\begin{array}{r}
\text { Maximize }\left\{Z(x)=\left(\frac{N_{1}(x)}{D_{1}(x)}, \frac{N_{2}(x)}{D_{2}(x)}, \ldots,\right.\right. \\
\\
\left.\left.\frac{N_{k}(x)}{D_{k}(x)}\right) \mid x \in X\right\},
\end{array}
$$

where $X=\left\{x \in R^{n} / A x \leq b, x \geq 0\right\}$ is convex and nonempty bounded set, $A$ is an $m \times n$ constraint matrix, $x$ is an $n$-dimensional vector of decision variable, and $b \in R^{m}$, $k \geq 2, N_{i}(x)=c_{i}^{T} x+\alpha_{i}, D_{i}(x)=d_{i}^{T} x+\beta_{i}$, for all $i=1, \ldots, k$, $c_{i}^{T}, d_{i}^{T} \in R^{n}, \alpha_{i}, \beta_{i} \in R$, for all $i=1, \ldots, k, D_{i}(x)=d_{i} x+\beta_{i}>$ 0 , for all $i=1, \ldots, k$, for all $x \in X$.

Definition 4. $\bar{x} \in R^{n}$ is an efficient solution of MOLFP if there is no $\widehat{x} \in R^{n}$ such that $N_{i}(\hat{x}) / D_{i}(\widehat{x}) \geq N_{i}(\bar{x}) / D_{i}(\bar{x})$, $i=1,2, \ldots, k, N_{i}(\hat{x}) / D_{i}(\hat{x})>N_{i}(\bar{x}) / D_{i}(\bar{x})$, for at least one $i$.

In this study, in order to solve MOLFP problem in (3), we can maximize each objective function $Z_{i}(x)$ subject to the given set of constraints using one of the methods proposed for single fractional objective function in [27] or others. Let $x_{i}^{*}$ and $Z_{i}^{*}$ be the global maximum points and values of each objective function $\operatorname{Max}\left\{Z_{i}(x)=\left(c_{i} x+\alpha_{i}\right) /\left(d_{i} x+\beta_{i}\right) \mid x \in\right.$ $X\}$ for all $i=1,2, \ldots, k$. Now, we can prove that the solution $\bar{x}$ is an efficient solution of $\operatorname{Max}\left\{Z_{i}(x)=\left(c_{i} x+\alpha_{i}\right) /\left(d_{i} x+\beta_{i}\right), i=\right.$ $1,2, \ldots, k \mid x \in X\}$.

If $\bar{x}$ is an optimal solution of problem: $\operatorname{Max}\left\{\sum_{i=1}^{k}\left(N_{i}(x)-\right.\right.$ $\left.\left.Z_{i}^{*} D_{i}(x)\right), \mid x \in X\right\}$, where is $Z_{i}^{*}=N_{i}\left(x_{i}^{*}\right) / D_{i}\left(x_{i}^{*}\right)$ for all $i=1,2, \ldots, k$.

Let $\bar{x}$ maximise problem $\operatorname{Max}\left\{\sum_{i=1}^{k}\left(N_{i}(x)-Z_{i}^{*} D_{i}(x)\right)\right.$ | $x \in X\}$; then we can write inequality $\sum_{i=1}^{k}\left(N_{i}(x)-Z_{i}^{*} D_{i}(x)\right) \leq$ $\sum_{i=1}^{k}\left(N_{i}(\bar{x})-Z_{i}^{*} D_{i}(\bar{x})\right)$ for any feasible solution $x \in X$. Hence,

$$
\begin{aligned}
\sum_{i=1}^{k}\left(N_{i}(x)-Z_{i}^{*} D_{i}(x)\right) & \leq \sum_{i=1}^{k}\left(N_{i}(\bar{x})-Z_{i}^{*} D_{i}(\bar{x})\right) \\
& \leq \sum_{i=1}^{k} \max \left\{N_{i}(x)-Z_{i}^{*} D_{i}(x)\right\} \\
& \leq \sum_{i=1}^{k}\left(N_{i}\left(x_{i}^{*}\right)-Z_{i}^{*} D_{i}\left(x_{i}^{*}\right)\right)=0
\end{aligned}
$$

for $x \in X$. 
From these inequalities, one obtains $N_{i}(x)-Z_{i}^{*} D_{i}(x) \leq$ $N_{i}(\bar{x})-Z_{i}^{*} D_{i}(\bar{x}) \leq 0$, for all $i, x \in X$.

We have $D_{i}(x)\left[N_{i}(x) / D_{i}(x)-Z_{i}^{*}\right] \leq D_{i}(\bar{x})\left[N_{i}(\bar{x}) / D_{i}(\bar{x})-\right.$ $\left.Z_{i}^{*}\right]$ for all $i$ :

$$
\left[\frac{N_{i}(x)}{D_{i}(x)}-Z_{i}^{*}\right] \leq \frac{D_{i}(\bar{x})}{D_{i}(x)}\left[\frac{N_{i}(\bar{x})}{D_{i}(\bar{x})}-Z_{i}^{*}\right]
$$

Both via Theorem 3 and the inequality $D_{i}(\bar{x}) / D_{i}(x) \geq 1$, one can write that $\left[N_{i}(x) / D_{i}(x)-Z_{i}^{*}\right] \leq\left[N_{i}(\bar{x}) / D_{i}(\bar{x})-\right.$ $\left.Z_{i}^{*}\right]$ and $\left[N_{i}(x) / D_{i}(x)\right] \leq\left[N_{i}(\bar{x}) / D_{i}(\bar{x})\right]$ for all $i$. If $\bar{x}$ maximise the problem $\operatorname{Max}\left\{\sum_{i=1}^{k}\left(N_{i}(x)-Z_{i}^{*} D_{i}(x)\right) \mid x \in X\right\}$, then it is an efficient solution of $\operatorname{Max}\left\{Z_{i}(x)=\left(c_{i} x+\alpha_{i}\right) /\left(d_{i} x+\right.\right.$ $\left.\left.\beta_{i}\right), i=1,2, \ldots, k \mid x \in X\right\}$. Now, assume that $\bar{x}$ is not an efficient of MOLFPP; then there exists a feasible solution $x$ of MOLFPP and $N_{i}(\bar{x}) / D_{i}(\bar{x}) \leq N_{i}(x) / D_{i}(x)$ for all $i$ and $N_{j}(\bar{x}) / D_{i j}(\bar{x})<N_{j}(x) / D_{j}(x)$ at least one $j$, where $x, \bar{x} \in X$. It follows that $N_{i}(\bar{x}) / D_{i}(\bar{x}) \leq N_{i}(x) / D_{i}(x) \leq Z^{*}, N_{i}(\bar{x})-$ $Z_{i}^{*} D_{i}(\bar{x}) \leq N_{i}(x)-Z_{i}^{*} D_{i}(x)$ for all $i$ and $N_{j}(\bar{x})-Z_{j}^{*} D_{i}(\bar{x})<$ $N_{j}(x)-Z_{i}^{*} D_{i}(x)$ at least one $j$. Summing the $k$-inequalities, we have $\sum_{i=1}^{k}\left(N_{i}(x)-Z_{i}^{*} D_{i}(x)\right) \leq \sum_{i=1}^{k}\left(N_{i}(\bar{x})-Z_{i}^{*} D_{i}(\bar{x})\right)$. This inequality leads to a contradiction.

Thus, we have made a proposal for the solution of MOLFP based on the above proof. These examples considered by Chakraborty and Gupta in [22] use Zimmermann's min operator for the fuzzy model.

\section{Numerical Examples}

Example 1. Let us consider a MOLFPP with two objectives as follows:

$$
\begin{array}{ll}
\operatorname{Max} & \left\{Z_{1}(x)=\frac{-3 x_{1}+2 x_{2}}{x_{1}+x_{2}+3}, Z_{2}(x)=\frac{7 x_{1}+x_{2}}{5 x_{1}+2 x_{2}+1}\right\} \\
\text { s.t. } & x_{1}-x_{2} \geq 1 \\
& 2 x_{1}+3 x_{2} \leq 15 \\
& x_{1} \geq 3 \\
& x_{1}, x_{2} \geq 0 .
\end{array}
$$

It is observed that $Z_{1}<0, Z_{2} \geq 0$, for each $x$ in the feasible region:

$$
\frac{-15}{7} \leq Z_{1} \leq \frac{-14}{23}, \quad \frac{139}{121} \leq Z_{2} \leq \frac{105}{77}
$$

This MOLFPP is equivalent to the following LPP. The given MOLFP problem can be written as

$$
\begin{array}{ll}
\operatorname{Max} \quad & \left\{-3 x_{1}+2 x_{2}+\frac{14}{23}\left(x_{1}+x_{2}+3\right)\right. \\
& \left.+7 x_{1}+x_{2}-\frac{105}{77}\left(5 x_{1}+2 x_{2}+1\right)\right\}, \\
\text { s.t. } \quad & x_{1}-x_{2} \geq 1, \\
& 2 x_{1}+3 x_{2} \leq 15, \\
& x_{1} \geq 3, \\
& x_{1}, x_{2} \geq 0 .
\end{array}
$$

The solution of the ealier linear programming problem is obtained as $x_{1}=3, x_{2}=2$.

The solution for original problem is given by

$$
x_{1}=3, \quad x_{2}=2, \quad Z_{1}=\frac{-5}{8}, \quad Z_{2}=\frac{23}{20} .
$$

Example 2. Let us consider a MOLFPP with three objectives as follows:

$$
\begin{aligned}
\operatorname{Max}\left\{Z_{1}(x)\right. & =\frac{-3 x_{1}+2 x_{2}}{x_{1}+x_{2}+3}, \\
Z_{2}(x) & =\frac{7 x_{1}+x_{2}}{5 x_{1}+2 x_{2}+1}, \\
Z_{3}(x) & \left.=\frac{x_{1}+4 x_{2}}{2 x_{1}+3 x_{2}+2}\right\},
\end{aligned}
$$

s.t. $x_{1}-x_{2} \geq 1$,

$$
\begin{aligned}
& 2 x_{1}+3 x_{2} \leq 15, \\
& x_{1}+9 x_{2} \geq 9, \\
& x_{1} \geq 3, \\
& x_{1}, x_{2} \geq 0 .
\end{aligned}
$$

It is observed that $Z_{1}<0, Z_{2} \geq 0, Z_{3} \geq 0$ for each $x$ in the feasible region. These values are $-53 / 26 \leq Z_{1} \leq-14 / 23$, $139 / 121 \leq Z_{2} \leq 23 / 17$, and $8 / 17 \leq Z_{3} \leq 14 / 17$.

The earlier MOLFP problem is equivalent to the following LP problem:

$$
\begin{aligned}
\operatorname{Max} \quad\{ & -3 x_{1}+2 x_{2}-\frac{14}{23}\left(x_{1}+x_{2}+3\right) \\
& +7 x_{1}+x_{2}-\frac{23}{17}\left(5 x_{1}+2 x_{2}+1\right)+x_{1} \\
& \left.+4 x_{2}-\frac{14}{17}\left(2 x_{1}+3 x_{2}+2\right)\right\},
\end{aligned}
$$

$$
\begin{array}{ll}
\text { s.t. } & x_{1}-x_{2} \geq 1 \\
& 2 x_{1}+3 x_{2} \leq 15 \\
& x_{1}+9 x_{2} \geq 9 \\
& x_{1} \geq 3 .
\end{array}
$$


The solution of the above linear programming problem is obtained as $x_{1}=3, x_{2}=2$. The solution for the given MOLFP problem is given by

$$
x_{1}=3, \quad x_{2}=2, \quad Z_{1}=\frac{-5}{8}, \quad Z_{2}=\frac{23}{20}, \quad Z_{3}=\frac{11}{14} .
$$

\section{Conclusion}

In this paper, we have presented a new solution to the Multiobjective Linear Fractional Programming Problem (MOLFPP). The solution is based on a theorem proposed in [7] dealing with nonlinear fractional programming with single objective function. With the help of this suggested approach, all of linear fractional objective functions of MOLFP problem become a single objective function. Furthermore the MOLFP problem is transformed into LPP. Thus, the complexity and the computations in solving MOLFP problem reduce in a certain amount. We used two numerical examples solved with different methods in $[18,22,28]$.

\section{Conflict of Interests}

The author declares that there is no conflict of interests regarding the publication of this paper.

\section{References}

[1] Y.-J. Lai and C. L. Hwang, Fuzzy Multiple Objective Decision Making, Springer, 1994.

[2] I. M. Stancu-Minasian and B. Pop, "On a fuzzy set approach to solving multiple objective linear fractional programming problem," Fuzzy Sets and Systems, vol. 134, no. 3, pp. 397-405, 2003.

[3] D. S. Kim, C. L. Jo, and G. M. Lee, "Optimality and duality for multiobjective fractional programming involving $n$-set functions," Journal of Mathematical Analysis and Applications, vol. 224, no. 1, pp. 1-13, 1998.

[4] J.-C. Liu and K. Yokoyama, " $\epsilon$-optimality and duality for multiobjective fractional programming," Computers \& Mathematics with Applications, vol. 37, no. 8, pp. 119-128, 1999.

[5] S. Tigan and I. M. Stancu-Minasian, “On Rohn's relative sensitivity coefficient of the optimal value for a linear-fractional program," Academy of Sciences of the Czech Republic. Mathematical Institute. Mathematica Bohemica, vol. 125, no. 2, pp. 227234, 2000.

[6] R. Patel, "Mixed-type duality for multiobjective fractional variational control problems," International Journal of Mathematics and Mathematical Sciences, no. 1, pp. 109-124, 2005.

[7] W. Dinkelbach, "On nonlinear fractional programming," Management Science, vol. 13, pp. 492-498, 1967.

[8] M. T. Arévalo, A. M. Mármol, and A. Zapata, "The tolerance approach in multiobjective linear fractional programming," Top, vol. 5, no. 2, pp. 241-252, 1997.

[9] H. I. Calvete and C. Gale, "The bilevel linear/linear fractional programming problem," European Journal of Operational Research, vol. 114, pp. 188-197, 1999.

[10] S. R. Yadav and R. N. Mukherjee, "Duality for fractional minimax programming problems," Australian Mathematical Society Journal B, vol. 31, no. 4, pp. 484-492, 1990.
[11] M. Sakawa, I. Nishizaki, and Y. Uemura, "Interactive fuzzy programming for two-level linear fractional programming problems with fuzzy parameters," Fuzzy Sets and Systems, vol. 115, no. 1, pp. 93-103, 2000.

[12] M. Sakawa and I. Nishizaki, "Interactive fuzzy programming for two-level linear fractional programming problems," Fuzzy Sets and Systems, vol. 119, no. 1, pp. 31-40, 2001.

[13] P. Gupta and D. Bhatia, "Sensitivity analysis in fuzzy multiobjective linear fractional programming problem," Fuzzy Sets and Systems, vol. 122, no. 2, pp. 229-236, 2001.

[14] O. M. Saad, "An iterative goal programming approach for solving fuzzy multiobjective integer linear programming problems," Applied Mathematics and Computation, vol. 170, no. 1, pp. 216225, 2005.

[15] J. B. G. Frenk and S. Schaible, "Fractional Programming," ERIM Report Series, Ref. No.ERS-2004-074-LIS., 2004.

[16] S. İ. Birbil, J. B. G. Frenk, and S. Zhang, "Generalized Fractional Programming with User Iteraction,” ERIM Report Series, Ref.No. ERS-2004-033-LIS., 2004.

[17] C. Mohan and H. T. Nguyen, "An interactive satisficing method for solving multiobjective mixed fuzzy-stochastic programming problems," Fuzzy Sets and Systems, vol. 117, no. 1, pp. 61-79, 2001.

[18] N. Güzel and M. Sivri, "Proposal of a solution to multi objective linear fractional programming problem," Sigma Journal of Engineering and Natural Sciences, vol. 2, pp. 43-50, 2005.

[19] M. Leber, L. Kaderali, A. Schönhuth, and R. Schrader, "A fractional programming approach to efficient DNA melting temperature calculation," Bioinformatics, vol. 21, no. 10, pp. 2375-2382, 2005.

[20] R. Jagannathan, "On some properties of programming problems in parametric form pertaining to fractional programming," Management Science, vol. 12, pp. 609-615, 1966.

[21] J. S. H. Kornbluth and R. E. Steuer, "Goal programming with linear fractional criteria," European Journal of Operational Research, vol. 8, no. 1, pp. 58-65, 1981.

[22] M. Chakraborty and S. Gupta, "Fuzzy mathematical programming for multi objective linear fractional programming problem," Fuzzy Sets and Systems, vol. 125, no. 3, pp. 335-342, 2002.

[23] M. K. Luhandjula, "Fuzzy approaches for multiple objective linear fractional optimization," Fuzzy Sets and Systems, vol. 13, no. 1, pp. 11-23, 1984.

[24] D. Dutta, R. N. Tiwari, and J. R. Rao, "Multiple objective linear fractional programming-a fuzzy set-theoretic approach," Fuzzy Sets and Systems, vol. 52, no. 1, pp. 39-45, 1992.

[25] B. I. Lee and D. W. Tcha, "An interactive procedure for fuzzy programming problems with linear fractional objectives," Computers and Industrial Engineering, vol. 16, pp. 269-275, 1989.

[26] S. Schaible, Analyse und Anwendungen von Quotientenprogrammen, Anton Hain, Meisenheim am Glan, Germany, 1978.

[27] A. Charnes and W. W. Cooper, "Programming with linear fractional functionals," Naval Research Logistics Quarterly, vol. 9, pp. 181-186, 1962.

[28] Y.-L. Chang and Q. S. B. Win, Version 1.0 for Windows, John Wiley \& Sons, 2001. 


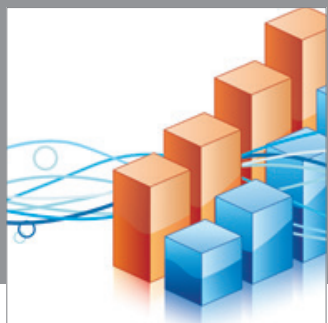

Advances in

Operations Research

mansans

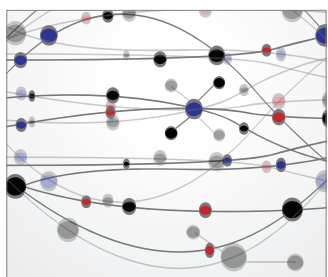

The Scientific World Journal
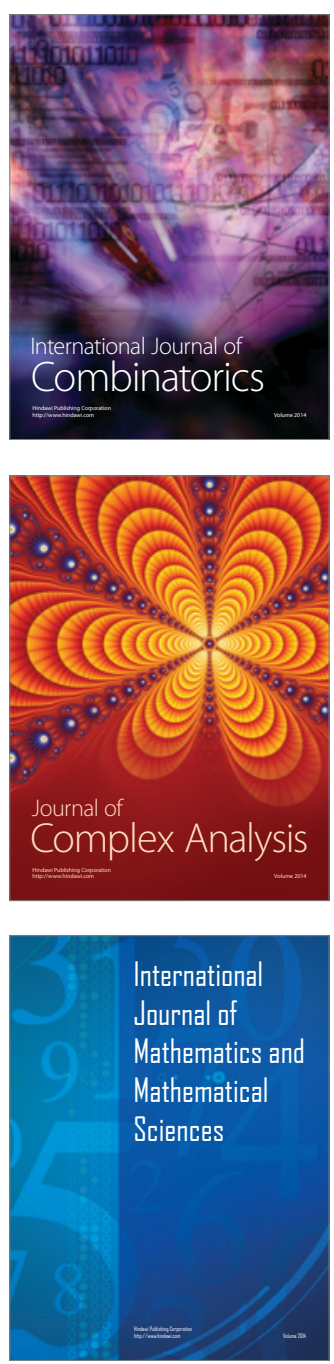
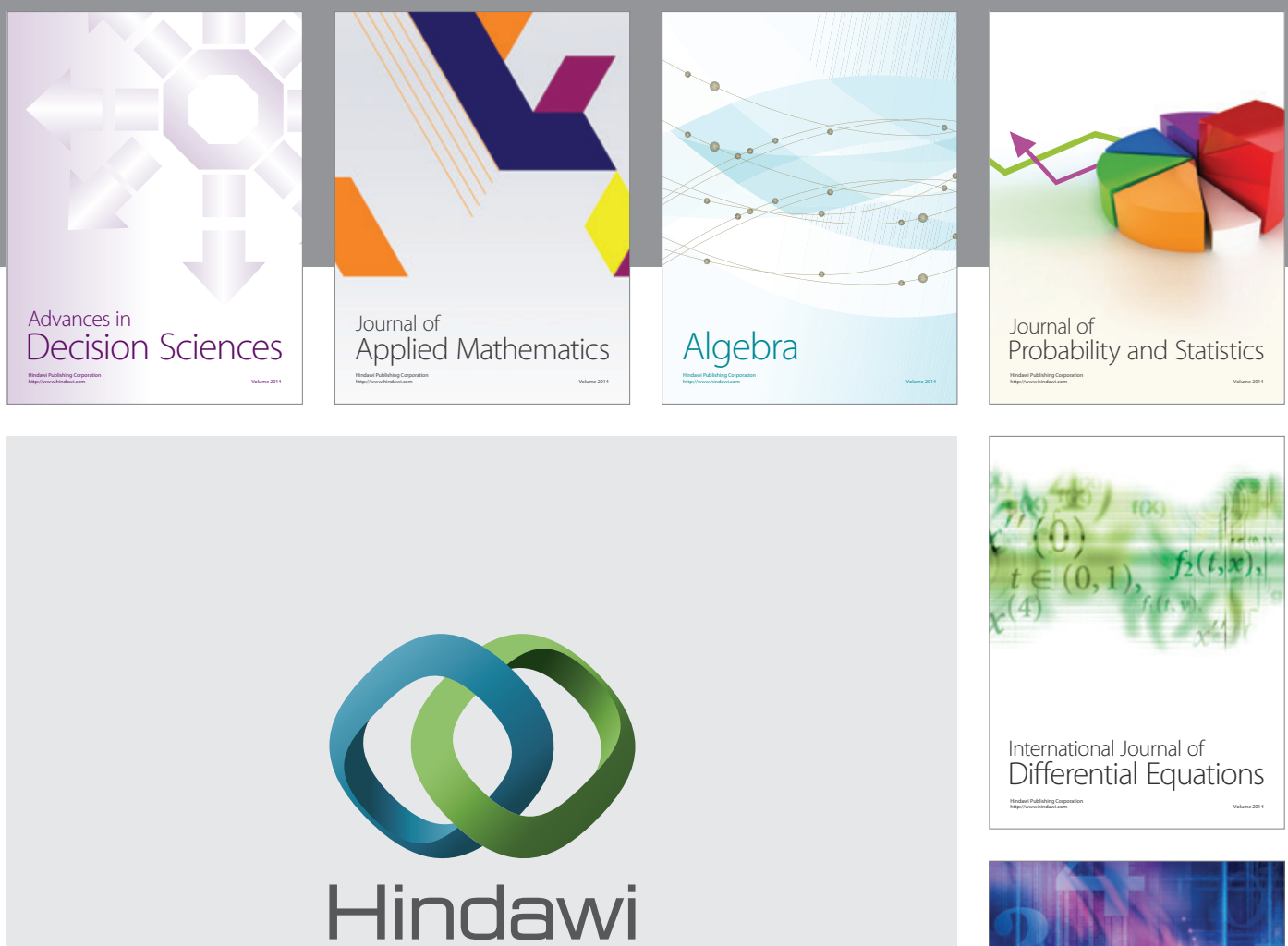

Submit your manuscripts at http://www.hindawi.com
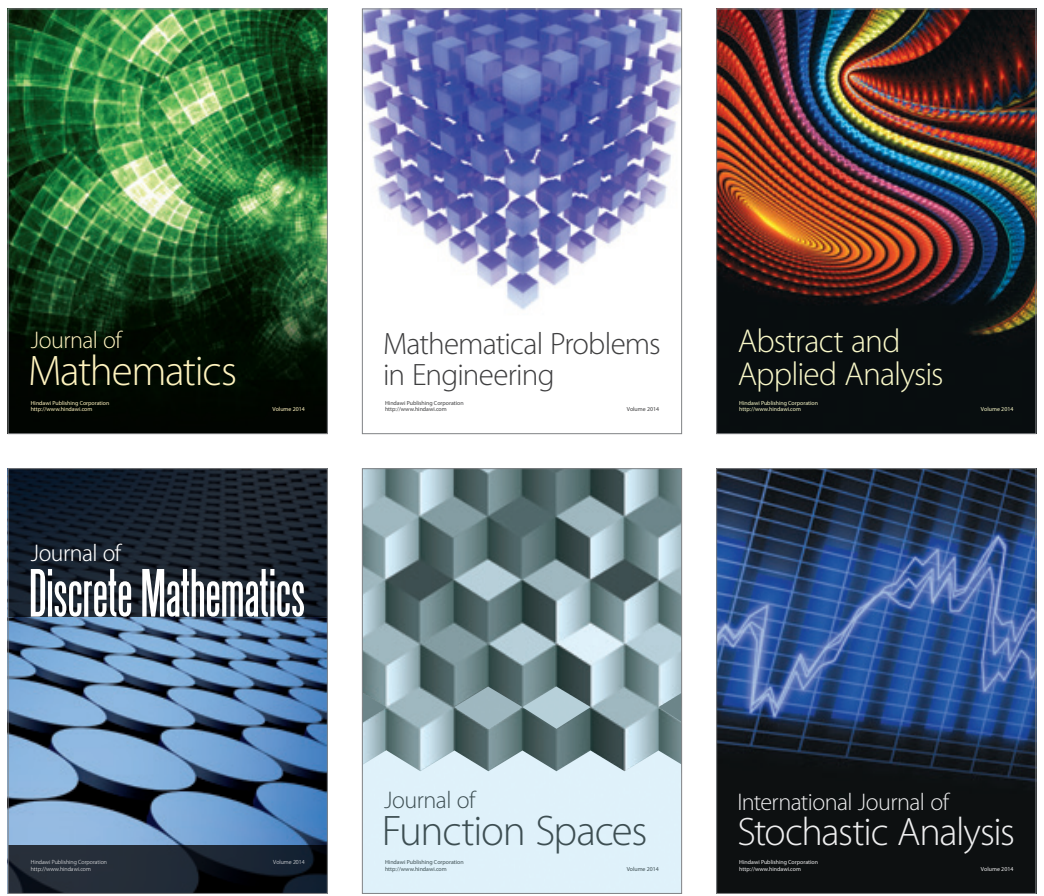

Journal of

Function Spaces

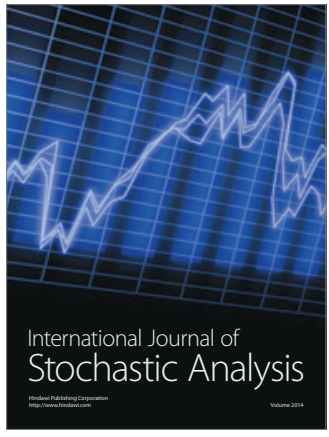

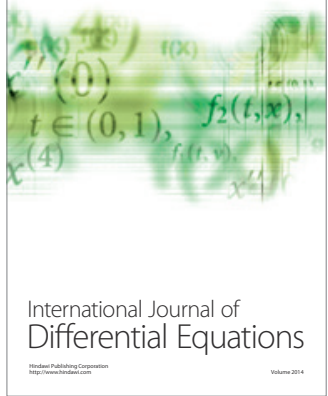
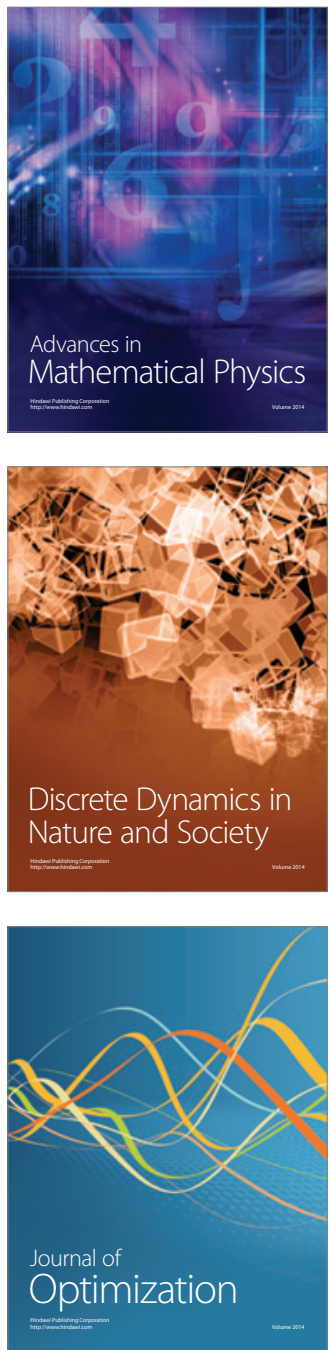